\title{
NILPOTENCY OF DERIVATIONS IN PRIME RINGS
}

\author{
DAVID W. JENSEN
}

(Communicated by Ken Goodearl)

\begin{abstract}
In 1957, E. C. Posner proved that if $\lambda$ and $\delta$ are derivations of a prime ring $R$, characteristic $R \neq 2$, then $\lambda \delta=0$ implies either $\lambda=0$ or $\delta=0$. We extend this well-known result by showing that, without any characteristic restriction, $\lambda \delta^{m}=0$ implies either $\lambda=0$ or $\delta^{4 m-1}=0$. We also prove that $\lambda^{n} \delta=0$ implies either $\delta^{2}=0$ or $\lambda^{12 n-9}=0$. In the case where $\lambda^{n} \delta^{m}=0$, we show that if $\lambda$ and $\delta$ commute, then at least one of the derivations must be nilpotent.
\end{abstract}

A derivation of a ring $R$ is an additive map $\lambda: R \rightarrow R$ satisfying $\lambda(x y)=$ $\lambda x \cdot y+x \cdot \lambda y$ for all $x, y \in R$. In 1957, Edward Posner showed that if $\lambda$ and $\delta$ are derivations of a prime ring $R$, characteristic $R \neq 2$, then $\lambda \delta=0$ implies either $\lambda=0$ or $\delta=0$ (see [3]). In this paper we show that in a prime ring with no characteristic restriction, $\lambda \delta=0$ implies either $\lambda=0$ or $\delta^{2}=0$. We generalize this result by proving that for any positive integer $m, \lambda \delta^{m}=0$ implies either $\lambda=0$ or $\delta^{4 m-1}=0$. Furthermore, we show that for any positive integer $n, \lambda^{n} \delta=0$ implies either $\delta^{2}=0$ or $\lambda^{12 n-9}=0$. Lastly, in the general case where $\lambda^{n} \delta^{m}=0$, we prove that if $\lambda$ and $\delta$ commute, then at least one of the derivations must be nilpotent.

Lemma 1. Assume $\lambda$ is a derivation of a prime ring $R$ and $\exists a \in R, a \neq 0$, such that $a\left(\lambda^{n} R\right)=0$ or $\left(\lambda^{n} R\right) a=0$ Then $\lambda^{2 n-1}=0$.

Proof. Start by assuming $a\left(\lambda^{n} R\right)=0$. Then for all $x, y \in R$, we have

$$
a \lambda^{n}(x y)=a\left(\sum_{i=0}^{n}\left(\begin{array}{c}
n \\
i
\end{array}\right) \lambda^{i} x \lambda^{n-i} y\right)=0 .
$$

Note that replacing $x$ by $\lambda^{n-1} x$ in (1) yields $a \lambda^{n-1} x \lambda^{n} y=0$. Replacing $x$ by $\lambda^{n-2} x$ and $y$ by $\lambda y$ in (1), and using the fact that $a \lambda^{n-1} x \lambda^{n} y=0$, we get $a \lambda^{n-2} x \lambda^{n+1} y=0$. At the next iteration we replace $x$ by $\lambda^{n-3} x$ and $y$ by $\lambda^{2} y$ in (1), and use $a \lambda^{n-1} x \lambda^{n} y=0$ and $a \lambda^{n-2} x \lambda^{n+1} y=0$, to get $a \lambda^{n-3} x \lambda^{n+2} y=0$. Continuing this process we eventually obtain $a x \lambda^{2 n-1} y=0$. Since this is true for all $x$ and $y$ in $R$, and $R$ is a prime ring, we conclude that $\lambda^{2 n-1}=0$. Similarly, if we begin with $\left(\lambda^{n} R\right) a=0$, we reach the same conclusion.

Received by the editors January 19, 1994; presented at the 96th Summer Meeting of the American Mathematical Society, Minneapolis, MN, August 15, 1994.

1991 Mathematics Subject Classification. Primary 16N60.

Key words and phrases. Derivations, nilpotency, prime rings. 
Lemma 2. If $\lambda$ and $\delta$ are derivations of a prime ring $R$ and $\lambda \delta=0$, then either $\lambda=0$ or $\delta^{2}=0$.

Proof. For all $x, y \in R$, we have $\lambda \delta(x y)=\lambda x \delta y+\delta x \lambda y=0$. Replacing $x$ by $\delta x$ yields $\delta^{2} x \lambda y=0$. Apply Lemma 1 .

The distinction between Posner's result and Lemma 2 is made clear by a simple example. A derivation $\lambda: R \rightarrow R$ is called an inner derivation if there exists $a \in R$ such that $\lambda(x)=[a, x]=a x-x a$, for all $x \in R$. Let $S$ be the $2 \times 2$ matrix ring over the Galois field $\left\{0,1, w, w^{2}\right\}$, with inner derivations $\lambda$ and $\delta$ defined by

$$
\lambda(X)=\left[\left(\begin{array}{ll}
0 & 1 \\
0 & 0
\end{array}\right), X\right] \quad \text { and } \delta(X)=\left[\left(\begin{array}{cc}
0 & w \\
0 & 0
\end{array}\right), X\right], \quad \forall X \in S .
$$

The characteristic of $S$ is 2 , and we have $\lambda \neq 0, \delta \neq 0, \lambda \delta=0$, and $\delta^{2}=0$.

Next we consider what can be said when $\lambda$ and $\delta$ are derivations of a prime ring $R$ and $\lambda^{n} \delta^{m}=0$. The following three theorems assure us that at least one of the derivations must be nilpotent when $m=1$, when $n=1$, or when $\lambda$ and $\delta$ commute.

Theorem 1. Let $\lambda$ and $\delta$ be derivations of a prime ring $R$, and let $\lambda \delta^{m}=0$ where $m$ is a positive integer. Then either $\lambda=0$ or $\delta^{r}=0$ where $r \leq 4 m-1$.

Proof. We proceed by induction, noting that Lemma 2 implies the result is true for $m=1$. Assume the theorem is true for $m=1,2,3, \ldots, k-1$. If $\lambda \delta^{k}=0$, then for all $x$ and $y$ in $R, \lambda \delta^{k}(x y)=\lambda\left(\sum_{i=0}^{k}\left(\begin{array}{c}k \\ i\end{array}\right) \delta^{k-i} x \delta^{i} y\right)=0$. Replacing $x$ by $\delta^{k-1} x$ and $y$ by $\delta^{k} y$ yields $\lambda\left(\delta^{k-1} x \delta^{2 k} y\right)=\lambda \delta^{k-1} x \delta^{2 k} y=0$. Applying Lemma 1 gives the desired result.

Theorem 2. Let $\lambda$ and $\delta$ be derivations of a prime ring $R$, and let $\lambda^{n} \delta=0$ where $n$ is a positive integer. Then either $\delta^{2}=0$ or $\lambda^{t}=0$ where $t \leq 12 n-9$.

Proof. The derivations of $R$ form a Lie ring under commutation (see [2, p. 4]). Therefore $[\delta, \lambda]=\delta \lambda-\lambda \delta$ is a derivation, $[\delta \lambda-\lambda \delta, \lambda]=\delta \lambda^{2}-2 \lambda \delta \lambda+\lambda^{2} \delta$ is a derivation, and $\left[\delta \lambda^{2}-2 \lambda \delta \lambda+\lambda^{2} \delta, \lambda\right]=\delta \lambda^{3}-3 \lambda \delta \lambda^{2}+3 \lambda^{2} \delta \lambda-\lambda^{3} \delta$ is a derivation. Continuing we may conclude that $\sum_{i=0}^{2 n-1}\left(\begin{array}{c}2 n-1 \\ i\end{array}\right)(-1)^{i} \lambda^{i} \delta \lambda^{2 n-1-i}$ is a derivation. The coefficients are not germane to the rest of the proof, so we suppress them from here on out. Using the fact that $\lambda^{n} \delta=0$ we get $\delta \lambda^{2 n-1}+\lambda \delta \lambda^{2 n-2}+\cdots+\lambda^{n-1} \delta \lambda^{n}$ is a derivation. Applying Lemma 2 to $\left(\delta \lambda^{2 n-1}+\lambda \delta \lambda^{2 n-2}+\cdots+\lambda^{n-1} \delta \lambda^{n}\right) \delta=0$ yields $\delta^{2}=0$ or

$$
\delta \lambda^{2 n-1}+\lambda \delta \lambda^{2 n-2}+\cdots+\lambda^{n-1} \delta \lambda^{n}=0 .
$$

If $\delta^{2} \neq 0$, then premultiply (2) by $\lambda^{n-1}$ to obtain $\lambda^{n-1} \delta \lambda^{2 n-1}=0$. Premultiplying (2) by $\lambda^{n-2}$ it follows that

$$
\begin{aligned}
& \lambda^{n-2} \delta \lambda^{2 n-1}+\lambda^{n-1} \delta \lambda^{2 n-2}=0 \\
& \quad \Rightarrow\left(\lambda^{n-2} \delta \lambda^{2 n-1}+\lambda^{n-1} \delta \lambda^{2 n-2}\right) \lambda=0 \\
& \quad \Rightarrow \lambda^{n-2} \delta \lambda^{2 n}=0 .
\end{aligned}
$$


Premultiplying (2) by $\lambda^{n-3}$ it follows that

$$
\begin{aligned}
& \lambda^{n-3} \delta \lambda^{2 n-1}+\lambda^{n-2} \delta \lambda^{2 n-2}+\lambda^{n-1} \delta \lambda^{2 n-3}=0 \\
& \quad \Rightarrow\left(\lambda^{n-3} \delta \lambda^{2 n-1}+\lambda^{n-2} \delta \lambda^{2 n-2}+\lambda^{n-1} \delta \lambda^{2 n-3}\right) \lambda^{2}=0 \\
& \quad \Rightarrow \lambda^{n-3} \delta \lambda^{2 n+1}=0
\end{aligned}
$$

Continuing this algorithm we eventually arrive at $\delta \lambda^{3 n-2}=0$. Applying Theorem 1 finishes the proof.

Lung Chung and Jiang Luh have shown that in a prime ring with characteristic 2 , the nilpotency of a nilpotent derivation must be of the form $2^{k}$ (see [1]). Therefore, when $R$ is not 2-torsion free, the possible values for nilpotency in Theorems 1 and 2 are further limited. For example, if we assume in Theorem 2 that characteristic $R=2, \lambda^{6} \delta=0$, and $\delta^{2} \neq 0$, then the nilpotency of $\lambda$ must be $1,2,4,8,16$, or 32 .

Theorem 3. Assume $\lambda$ and $\delta$ are derivations of a prime ring $R$, and assume $\lambda^{n} \delta^{m}=0$ where $n$ and $m$ are positive integers. If $\lambda$ and $\delta$ commute, then at least one of them is nilpotent.

Proof. From the hypotheses we know that for all $x, y \in R$,

$$
\begin{gathered}
\lambda^{n} \delta^{m}\left(\delta^{m-1} \lambda^{n} x \lambda^{n-1} y\right)=\lambda^{n}\left(\delta^{m-1} \lambda^{n} x \delta^{m} \lambda^{n-1} y\right)=\delta^{m-1} \lambda^{2 n} x \delta^{m} \lambda^{n-1} y=0 \\
\Rightarrow \lambda^{n} \delta^{m}\left(\delta^{m-2} \lambda^{2 n} x \delta^{m} \lambda^{n-1} y\right)=\lambda^{n}\left(\delta^{m-2} \lambda^{2 n} x \delta^{2 m} \lambda^{n-1} y\right) \\
=\delta^{m-2} \lambda^{3 n} x \delta^{2 m} \lambda^{n-1} y=0 \\
\Rightarrow \lambda^{n} \delta^{m}\left(\delta^{m-3} \lambda^{3 n} x \delta^{2 m} \lambda^{n-1} y\right)=\lambda^{n}\left(\delta^{m-3} \lambda^{3 n} x \delta^{3 m} \lambda^{n-1} y\right) \\
=\delta^{m-3} \lambda^{4 n} x \delta^{3 m} \lambda^{n-1} y=0 \\
\vdots \quad \begin{array}{r}
\Rightarrow \lambda^{n} \delta^{m}\left(\delta \lambda^{(m-1) n} x \delta^{(m-2) m} \lambda^{n-1} y\right)=\lambda^{n}\left(\delta \lambda^{(m-1) n} x \delta^{(m-1) m} \lambda^{n-1} y\right) \\
=\delta \lambda^{m n} x \delta^{(m-1) m} \lambda^{n-1} y=0
\end{array} \\
\Rightarrow \lambda^{n} \delta^{m}\left(\lambda^{m n} x \delta^{(m-1) m} \lambda^{n-1} y\right)=\lambda^{n}\left(\lambda^{m n} x \delta^{m^{2}} \lambda^{n-1} y\right) \\
=\lambda^{(m+1) n} x \delta^{m^{2}} \lambda^{n-1} y=0
\end{gathered}
$$

Using Lemma 1 , we have $\lambda^{2(m+1) n-1}=0$ or $\lambda^{n-1} \delta^{m^{2}}=0$. If $\lambda^{2(m+1) n-1} \neq 0$, we then apply the above process to $\lambda^{n-1} \delta^{m^{2}}=0$ to get $\lambda^{2\left(m^{2}+1\right)(n-1)-1}=0$ or $\lambda^{n-2} \delta^{m^{4}}=0$. If $\lambda$ is not nilpotent, we continue and eventually arrive at $\delta^{m^{2^{n}}}=0$.

As a final note, if $\lambda, \delta$, and $\gamma$ are derivations of a prime ring $R$ and $\lambda^{n} \delta^{m} \gamma^{h}=0$, it does not follow that one of the derivations must be nilpotent. For example, let $T$ be the $3 \times 3$ matrix ring over a division ring $D$, and let $\lambda, \delta$, and $\gamma$ be the inner derivations defined by the unit matrices $E_{11}$, $E_{22}$, and $E_{33}$, respectively. Then the derivations commute with each other, and $\lambda \delta \gamma=0$. However, none of the derivations are nilpotent. 


\section{REFERENCES}

1. L. O. Chung and J. Luh, Nilpotency of derivations. II, Proc. Amer. Math. Soc. 91 (1984), 357-358.

2. I. Kaplansky, Lie algebras and locally compact groups, Univ. of Chicago Press, Chicago, 1974.

3. E. C. Posner, Derivations in prime rings, Proc. Amer. Math. Soc. 8 (1957), 1093-1100.

Department of Mathematical Sciences, United States Military Academy, West Point, New YoRK 10996

E-mail address: ad95880usma2.usma.edu 